Internal Medicine, Ann Arbor, United States of America; ${ }^{9}$ Novartis Pharma AG, Basel, Switzerland; ${ }^{10}$ Ospedale Pediatrico Bambino Gesù, Division of Rheumatology, Rome, Italy; ${ }^{11}$ Department of Pediatric Rheumatology $\mathrm{CHU}$ de Bicêtre, CEREMAIA, Le Kremlin Bicêtre, France

Background: Familial Mediterranean Fever (FMF) is a hereditary autoinflammatory disease associated with mutations in the MEFV gene. Colchicine is the cornerstone of current therapy for FMF; however, a subset of patients are resistant or intolerant to it. Previously published results from the CLUSTER trial [NCT02059291] demonstrated that canakinumab, a fully human anti-interleukin-1 $\beta$ monoclonal antibody, was effective in controlling and preventing flares in patients with colchicine-resistant familial Mediterranean fever (crFMF). ${ }^{1}$

Objectives: To evaluate the long-term efficacy and safety of canakinumab to treat patients with crFMF during Epoch 4 of the CLUSTER study.

Methods: Patients with active crFMF (baseline flare) were enrolled in the CLUSTER study. During Epoch 4 (weeks 40 to 113), patients received open-label canakinumab 150 or $300 \mathrm{mg}$, every 4 or 8 weeks (q4w or q8w). Patients started Epoch 4 on the same regimen that they were receiving at the end of Epoch 3, and stepwise up-titration of canakinumab was allowed in patients who experienced a flare, to a maximum dose of $300 \mathrm{mg} \mathrm{q4w}$. We evaluated disease activity every 8 weeks using the physician global assessment of disease activity (PGA), counting the number of flares (defined as $P G A \geq 2$ and $C R P>30 \mathrm{mg} / \mathrm{L}$ ), and measuring serum concentrations of $C$ reactive protein $(C R P)$ and serum amyloid A (SAA). Safety was assessed by the determination and classification of adverse events (AEs). We analysed safety and efficacy separately in two subgroups of patients receiving a cumulative dose of canakinumab lower than $2700 \mathrm{mg}$, or equal or higher than $2700 \mathrm{mg}$.

Results: Of the 61 patients with active crFMF who started the CLUSTER study, 60 entered Epoch 4 and 57 completed it. During the 72-week period, 35/60 $(58.3 \%)$ patients experienced no flares, and $23 / 60$ (38.3\%) had one single flare, as compared with a median of 17.5 flares per year reported at baseline. The incidence of flares was similar in the two cumulative dose groups. PGA scores indicated no disease activity for the majority of patients throughout the study, in both cumulative dose groups. $23 / 57(40 \%)$ of patients remained in the lower dosing group (150 mg q8w) until study end, whereas $9 / 57$ (16\%) required the highest dose allowed (300 mg q4w). Patients with higher body weight had an increased probability to require up-titration of canakinumab to control disease activity. Median CRP concentrations were lower than $10 \mathrm{mg} / \mathrm{L}$ at every time point in both cumulative dose groups, while median SAA concentrations remained in the $16-70 \mathrm{mg} / \mathrm{L}$ range, and were higher in the group receiving $\geq 2700 \mathrm{mg}$ canakinumab (Figure 1). No opportunistic infections, renal disease caused by amyloidosis, new or unexpected AEs were reported.

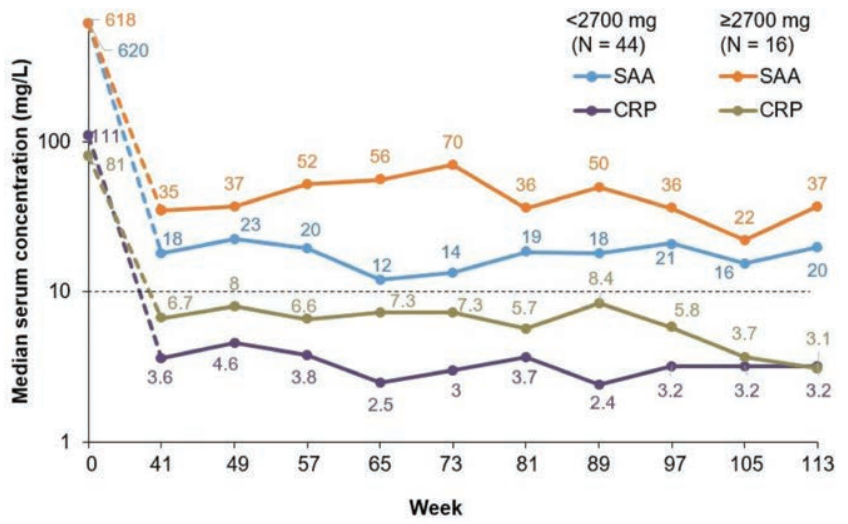

Figure 1. SAA and CRP blood levels in Epoch 4 of the CLUSTER study, in two subgroups of patients treated with a cumulative dose of canakinumab $<2700 \mathrm{mg}$ or $\geq 2700 \mathrm{mg}$

Conclusion: Patients with crFMF treated with canakinumab during 72 weeks experienced a minimal incidence of flares and good control of clinical disease activity, with no new safety signals reported.

References:

[1] De Benedetti F et al. N Engl J Med 2018;378:1908-19.

Disclosure of Interests: Seza Özen Consultant of: Novartis, Pfizer, Speakers bureau: SOBI, Novartis, Eldad Ben-Chetrit Speakers bureau: Novartis, Ivan Foeldvari Consultant of: Novartis, Gil Amarilyo Grant/research support from: Novartis, Speakers bureau: Novartis, Huri Ozdogan: None declared, Steven Vanderschueren: None declared, Katherine Marzan Grant/research support from: Novartis, J Michelle Kahlenberg Grant/research support from: Celgene, BMS, Consultant of: Eli Lilly, AstraZeneca, BMS, Boehringer Ingleheim, Elise Dekker Employee of: Novartis, Fabrizio De Benedetti Grant/research support from: AbbVie, Pfizer, Novartis, Novimmune, Sobi, Sanofi, Roche, Speakers bureau: AbbVie, Novartis, Roche, Sobi, Isabelle Koné-Paut Consultant of: Novartis, Chugai, Pfizer, LFB, AbbVie, Novimmune, SOBI

DOI: 10.1136/annrheumdis-2020-eular.522

\section{OP0273 \\ ADHERENCE TO COLCHICINE TREATMENT AND COLCHICINE RESISTANCE IN A MULTICENTRIC FMF NATIONAL COHORT}

R. Gallizzi ${ }^{1}$, M. Bustaffa ${ }^{1}$, F. Mazza ${ }^{1}$, D. Sutera ${ }^{1}$, G. Fabio ${ }^{1}$, L. Obici ${ }^{1}$,

M. Alessio ${ }^{1}$, D. Rigante ${ }^{1}$, L. Cantarini ${ }^{1}$, A. Insalaco ${ }^{1}$, M. Cattalini ${ }^{1}$, M.

C. Maggio ${ }^{1}$, G. Simonini ${ }^{1}$, A. N. Olivieri ${ }^{1}$, S. Pastore ${ }^{1}$, M. Lancieri ${ }^{1}$, N. Ruperto ${ }^{1}$, M. Gattorno'. ' ${ }^{1}$ on the behalf of the FMF Italian Study Group, Genova, Italy

Background: Colchicine is the standard treatment for Familiar Mediterranean Fever (FMF), however about $5 \%$ of patients experience colchicine resistance. There is no standard definition of colchicine resistance. Recently a panel of experts elaborated a new definition based on a Delphi consensus approach

Objectives: We aim to describe main features of the disease and clinical outcome of a cohort of FMF patients with particular interest on the colchicine resistance and tolerability according to the definitions proposed by the recent consensus.

Methods: Since November 2009, 425 Italian pediatric and adult FMF patients (pts) from 13 centers were enrolled in a national longitudinal cohort study, using the international EUROFEVER registry. Demographic, genetic and clinical data, including response to treatment, were analyzed. Supplementary information on health related quality of life and treatment adherence was also collected by a specific questionnaire.

Results: Complete information were available in 341 pts (189 M and 152 F, 211 children and 120 adults). The median age at disease onset was 5.0 years ( 1 $\mathrm{m}-59 \mathrm{y}$ ); the mean diagnostic delay was $8.7 \mathrm{y}$ (range 0-61 y). The median age at enrollment was $12.1 \mathrm{y}$ (range $3 \mathrm{~m}-82 \mathrm{y}$ ). The MEFV genotype was the following: $103(30.2 \%)$ pts carried biallelic pathogenic variants; 59 (17.3\%) one pathogenic variants and one VOUS /LB variant; 27 (7.9\%) had biallelic VOUS/LB variants; 97 $(28.45 \%)$ were heterozygous for pathogenic variants; 30 (8.8\%) were heterozygous for VOUS/LB, 25 (7.33\%) were genetically negative.

Colchicine treatment was used in 280 pts; during treatment, biologic treatment (anti-IL1) in 22 pts. 61 pts received NSAID or steroid on demand.

We analyzed the behavior of the pts treated with colchicine according to the statements on colchicine resistance/intolerance defined by Ozen et al (1) (Table 1)

\section{Table 1.}

\begin{tabular}{|c|c|}
\hline Adherence & $\begin{array}{l}62 \% \text { displayed a total adherence }(>90 \% \text { of prescription); } 10.8 \% \\
\text { a good adherence }(50-89 \% \text { of prescriptions); } 1.9 \% \text { poor } \\
\text { adherence }(<50 \% \text { of prescriptions); } 0.9 \% \text { no adherence }\end{array}$ \\
\hline \multirow{9}{*}{$\begin{array}{l}\text { Dose adjustment criteria/ } \\
\text { Recommended maxi- } \\
\text { mum colchicine dose }\end{array}$} & Mean colchicine dose: \\
\hline & Pts $<5$ years: $0.57 \mathrm{mg} / \mathrm{de}$ (std. dev. 0.18 ) \\
\hline & $5-10$ year: $0.77 \mathrm{mg} / \mathrm{die}(\mathrm{std} . \mathrm{dev} .0 .23)$ \\
\hline & $10-18$ years: $1.1 \mathrm{mg} /$ die (std. dev. 0.39 ) \\
\hline & Adults: $1.16 \mathrm{mg} / \mathrm{die}$ (std. dev. 0.37) \\
\hline & Pts with a dose inferior to the minimum recommended dose \\
\hline & $5-10$ years: $2.5 \%$ \\
\hline & $10-18$ years: $15 \%$ \\
\hline & Adults: $4 \%$ \\
\hline \multirow[t]{3}{*}{ Resistance to Colchicine } & $\begin{array}{l}\text { Resistance was be defined as persistence of fever attacks, } \\
\text { despite optimal treatment. } 41.6 \% \text { pts had a complete } \\
\text { disease control }\end{array}$ \\
\hline & $32.8 \%$ Pts had $<1$ episode/month for 3 months \\
\hline & $25.5 \%$ had $\geq 1$ episode/month for 3 months \\
\hline $\begin{array}{l}\text { Inclusion of secondary } \\
\text { amyloidosis in the } \\
\text { definition of colchicine } \\
\text { resistance }\end{array}$ & 5 adult pts (1.5\%) displayed amyloidosis \\
\hline Colchicine intolerance & 11 pts $(3.2 \%)$ withdraw colchicine because of drug intolerance \\
\hline $\begin{array}{l}\text { Patient quality of life } \\
\text { and patient-reported } \\
\text { outcomes }\end{array}$ & $\begin{array}{l}20.7 \% \text { of pts experience fatigue or chronic pain, } 16.9 \% \\
\text { limitations in daily activities, and } 16.9 \% \text { have lost school/ } \\
\text { work days. }\end{array}$ \\
\hline
\end{tabular}

Conclusion: Almost $58 \%$ of FMF pts display disease activity despite colchicine treatment. The treatment is generally under-dosed, especially in children. The adherence and the compliance to the treatment is generally good.

References:

[1] Ozen S et all. Recommendation on colchicine dosing and definition of colchicine resistance/intolerance in the management of FMF. Pediatric Rheumatology, 2019.

Acknowledgments: This research was financial supported by Novartis AG Disclosure of Interests: Romina Gallizzi: None declared, Marta Bustaffa: None declared, Francesca Mazza: None declared, Diana Sutera: None declared, 
Giovanna Fabio: None declared, Laura Obici: None declared, Maria Alessio: None declared, Donato Rigante: None declared, Luca Cantarini: None declared, Antonella Insalaco: None declared, Marco Cattalini: None declared, Maria Cristina Maggio: None declared, Gabriele Simonini: None declared, Alma Nunzia Olivieri: None declared, Serena Pastore: None declared, Maddalena Lancieri: None declared, Nicolino Ruperto Grant/research support from: Bristol-Myers Squibb, Eli Lily, F Hoffmann-La Roche, GlaxoSmithKline, Janssen, Novartis, Pfizer, Sobi (paid to institution), Consultant of: Ablynx, AbbVie, AstraZeneca-Medimmune, Biogen, Boehringer Ingelheim, Bristol-Myers Squibb, Eli Lily, EMD Serono, GlaxoSmithKline, Hoffmann-La Roche, Janssen, Merck, Novartis, Pfizer, R-Pharma, Sanofi, Servier, Sinergie, Sobi, Takeda, Speakers bureau: Ablynx, AbbVie, AstraZeneca-Medimmune, Biogen, Boehringer Ingelheim, Bristol-Myers Squibb, Eli Lily, EMD Serono, GlaxoSmithKline, Hoffmann-La Roche, Janssen, Merck, Novartis, Pfizer, R-Pharma, Sanofi, Servier, Sinergie, Sobi, Takeda, Marco Gattorno Consultant of: Sobi, Novartis, Speakers bureau: Sobi, Novartis

DOI: 10.1136/annrheumdis-2020-eular.1402

\section{OP0274 CLINICAL ASPECTS, LABORATORY CHARACTERISTICS AND TREATMENT RESPONSES OF AA AMYLOIDOSIS: SINGLE CENTER EXPERIENCE WITH 163 PATIENTS}

M. Bektas ${ }^{1}$, N. Koca ${ }^{1}$, E. Oguz2, B. Ince ${ }^{1}$, Y. Yalçınkaya ${ }^{1}$, B. Artim-Esen ${ }^{1}$, M. Inanc ${ }^{1}$, M. L. Ocal' ${ }^{1}$, A. Gül' ${ }^{1}{ }^{1}$ Istanbul Faculty of Medicine, Internal Medicine, Rheumatology Division, Istanbul, Turkey; ${ }^{1}$ Istanbul Faculty of Medicine, Internal Medicine, Rheumatology Division, Istanbul, Turkey

Background: AA amyloidosis has been associated with uncontrolled chronic inflammatory diseases such as rheumatoid arthritis (RA), ankylosing spondylitis (AS), inflammatory bowel disease (IBD) and hereditary periodic fever syndromes, and the most common cause is familial Mediterranean fever (FMF) in Turkey. Objectives: We herein aimed to evaluate clinical and laboratory characteristics and treatment responses of patients with AA amyloidosis retrospectively in a tertiary referral center.

Methods: Study group was consisting of patients with biopsy proven AA amyloidosis, and their data were recorded from their charts. Treatment responses were categorized as follows: complete response was defined as no increase in serum creatinine and a proteinuria below $1 \mathrm{gr} /$ day; partial response as $50 \%$ decrease in proteinuria; and stable disease as no significant change in serum creatinine and proteinuria. Progressive disease was defined as increase in serum creatinine and/or proteinuria under treatment.

Results: 173 patients were identified, and 10 patients with no biopsy result and/ or missing data were excluded. A total of 163 patients (79 females, 84 males) were included in the study. Median age of patients was 45.4 , and median age at diagnosis of amyloidosis was 33.5. Most common cause of amyloidosis was FMF $(78.5 \%)$, followed by idiopathic cases $(7.9 \%)$ and patients with AS (4.9\%). A quarter (26\%) of amyloidosis patients had a family history for AA amyloidosis, and $59 \%$ of patients with FMF had a family history of FMF. Amyloidosis was confirmed by renal biopsy in $76.1 \%$, by gastrointestinal (GIS) biopsy in $11.7 \%$, and by other biopsies in the remaining. Renal involvement was documented in 160 (98.2\%) patients, while GIS involvement in $20.9 \%$, heart in $13.5 \%$, thyroid in $3.7 \%$ and bone marrow in $3.1 \%$. In FMF patients, most common MEFV mutation was M694V (77.7\%); and $66.7 \%$ of the patients had homozygous, $14.6 \%$ had compound heterozygous, and $18.7 \%$ heterozygous exon 10 variants. Mean age at diagnosis of amyloidosis was earlier in homozygotes (29.1) and compound heterozygotes (32.3) compared to heterozygotes (43.9) $(p=0.001)$. There was no difference in treatment responses, organ involvement, progression to end stage renal disease (ESRD) and mortality between monoallelic and biallelic exon 10 mutations $(p=0.42)$. While $44.3 \%$ ( $n$ $=70$ ) of patients had chronic renal disease (CRD) at time of diagnosis, ESRD developed in $45.3 \%(n=73)$ of patients. During follow-up, 55 patients underwent renal transplantation and recurrence of renal amyloidosis occurred in $24 \%$ of them. Mean creatinine and proteinuria levels at time of diagnosis were higher in patients with ESRD than those without ESRD ( $p<0.001, p=0.03$ respectively). Progression to ESRD was significantly higher in patients with GFR $\leq 60 \mathrm{ml} / \mathrm{min}$ at time of admission ( $\% 14.5$ vs $\% 41.7, p=0.005$, Figure 1$)$. A total of $113(70.2 \%)$ patients used biological agents, most commonly used biological agent was anakinra $(n=81)$. Canakinumab was used in 17 and other biological agents in 17 patients. Complete response was observed in $49.1 \%$, partial response was observed in $6.2 \%$, and progressive progression was observed in $21.7 \%$. GIS and cardiac involvements were associated with progressive course $(p<0.001)$ and increased mortality $(p=$ $0.002, p<0.001$, respectively), and overall mortality rate was $8.7 \%$.

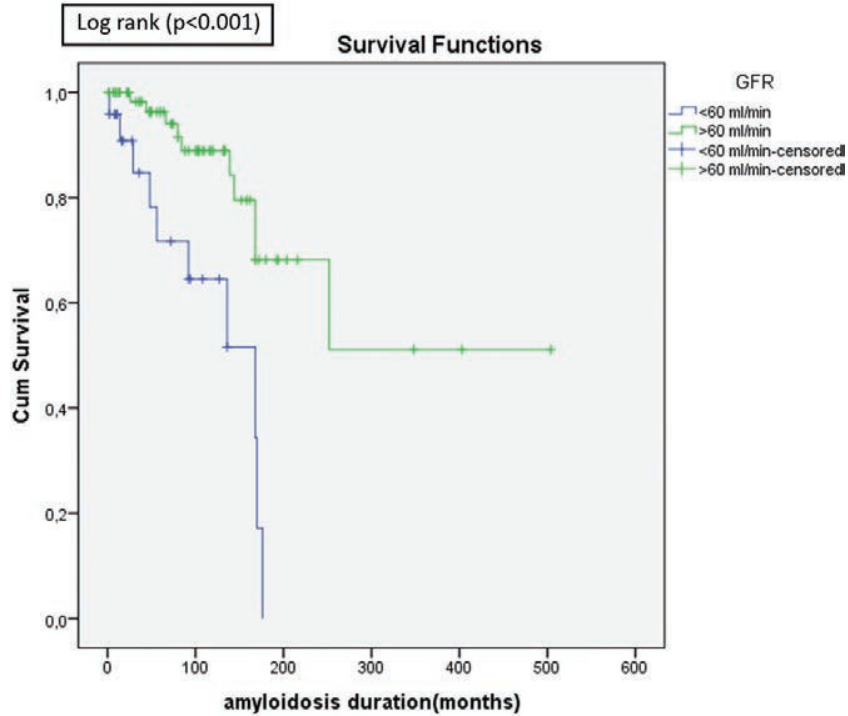

Figure 1 : Survival graphic of AA amyloidosis patients who developed ESRD according to their baseline GFR status

Conclusion: Increased rate of ESRD and progression of amyloidosis findings in patients who presented with GFR $<60 \mathrm{ml} / \mathrm{min}$ emphasize the importance of early diagnosis. Although mortality rate is very high in patients with $A A$ amyloidosis due to FMF disease, it may be possible to reduce mortality with an effective treatment. Disclosure of Interests: None declared

DOI: 10.1136/annrheumdis-2020-eular.3805

\section{OP0275 \\ REAL-WORLD CLINICAL BURDEN AND GLUCOCORTICOID USE IN PATIENTS WITH GIANT CELL ARTERITIS}

R. Punekar ${ }^{1}$, P. Lafontaine ${ }^{2}$, J. H. Stone ${ }^{3} .{ }^{1}$ Sanofi, Cambridge, United States of America; ${ }^{2}$ Sanofi, Bridgewater, United States of America; ${ }^{3}$ Harvard Medical School, Boston, United States of America

Background: Giant cell arteritis (GCA) is a rare form of vasculitis usually manifesting in people aged $\geq 50 \mathrm{yr}$ and is more common in women. Symptoms include headache, jaw claudication, fatigue, polymyalgia; and blindness if untreated. While risks of complications can be reduced with promptly administered highdose glucocorticoids (GC; $20-60 \mathrm{mg}$ for $2-4 \mathrm{wk}$, then slowly tapered), further risks of high GC exposure and related complications over the course of therapy remain.

Objectives: To compare GC use and GC-related complications in GCA patients (pts) vs a general population (GnP) cohort.

Methods: This retrospective, observational cohort study was based on Optum's de-identified Clinformatics ${ }^{\circledR}$ Data Mart Database (01/01/06-30/06/18, study period). The GCA cohort included pts with $\geq 1$ inpatient or $\geq 2$ outpatient claims $\geq 30$ days apart with GCA-related diagnosis codes (ICD-9: 446.5x/ICD-10: M31.6x) between 01/01/06-30/06/17 (pt identification period) during which first occurrence of a GCA-related medical claim was set as index date (ID). The GnP cohort included pts without any medical claims for rheumatoid arthritis, GCA or polymyalgia rheumatica diagnosis codes during the study period, with their ID set as 12 mo from start of continuous health plan enrollment. Pts in both cohorts were required to be age $\geq 50 \mathrm{yr}$ (on the ID) with continuous health plan enrollment $\geq 12$ mo pre- and post-ID. Cohorts were 1:1 propensity score matched. GC use and incidence of GC-related complications were assessed from GC initiation, starting from the baseline period (12-mo pre-ID) to the end of GC use during the post-index period (ie the end of data availability, end of the study period, or death, whichever occurred first). Descriptive analyses included mean, standard deviation (SD) and median values for continuous variables, and frequency ( $\mathrm{n}$ and \%) for categorical variables. Continuous variables were compared between matched cohorts using $t$-tests and Wilcoxon sum rank tests. Categorical variables were compared between matched cohorts using Chi-square tests or Fisher's exact tests. Duration of GC use was analyzed using the Kaplan-Meier method and compared between matched cohorts using log-rank tests.

Results: There were 6071 pts included in each of the GCA and matched GnP cohorts; median age per cohort was $76 \mathrm{yr}$, median Elixhauser comorbidity index score was 3.0, and the majority $(\sim 75 \%)$ were women. The median follow-up duration was 44 and 48 mo in the GCA and GnP cohorts, respectively. A higher proportion of pts in the GCA cohort than the GnP cohort ( 90.6 vs $63.8 \% ; p<0.001$ ) used GC. The mean (SD) duration of GC therapy was $230.5( \pm 326.8)$ days in 